\title{
Study of Microleakage at Class V Cavities Prepared by Er:YAG Laser Using Rewetting Surface Treatment
}

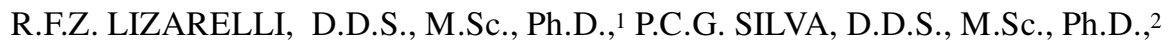 \\ S.T. PORTO NETO, D.D.S., M.Sc., Ph.D., ${ }^{2}$ and V.S. BAGNATO, M.Sc., APP, Ph.D. ${ }^{1}$
}

\begin{abstract}
Objective: This study was conducted to analyze microleakage in Class $\mathrm{V}$ cavity preparation, using rewetting (or not) just after burr or Er:YAG laser preparation of enamel and dentin walls in permanent teeth. Background Data: Several studies reported microleakage around composite restorations when cavity preparation was done or treated by Er:YAG laser. As the hybridized laser is removed when this laser is used to cut dental hard tissue, there is a need for new materials or techniques to minimize gaps and microleakage. Results: Primer solution showed significant effect in enamel and dentin, at the level of 5\%, when Er:YAG laser was used as a cutting tool. Using primer solution after phosphoric acid in preparations with the laser, microleakage was similar in degree to when cavities were prepared with the burr. Conclusion: Re-wetting surface just after Er:YAG irradiation and chemical treatment with phosphoric acid using HEMA aqueous solution seems to improve the quality of bioattachment between the adhesive system and enamel/dentin, showing similarities between restoration behaviors independently of the cutting tool, whether burr or laser.
\end{abstract}

\section{INTRODUCTION}

$\mathrm{T}$ HERE IS A CONSTANT NEED for materials or techniques that ensure adhesion to tooth structure to minimize the potential for leakage. The marginal adaptation of restorative materials is influenced by shrinkage from polymerization and a high coefficient of thermal expansion. ${ }^{1}$ The laser is now established as a suitable tool for the selective and precise removal of carious dental tissue. ${ }^{2-8}$ If correctly used, lasers minimize the loss of healthy tissue and promote comfort for patients during the procedure. The absence of noise and mechanical vibration results in reduced pain, making the procedure tolerable for most patients.

The interaction of Er:YAG laser and dental hard tissues obeys an explosive thermo-mechanical ablation mediated by water. ${ }^{9}$ Absorption of heat by water is the most likely mechanism of ablation of dental hard tissue at $2940 \mathrm{~nm}$. During rapid heating, the inertial confined water can create enormous subsurface pressures that lead to the explosive removal of the surrounding mineral matrix. This can occur at temperatures well below the melting point of the mineral phase of enamel. Several studies of hard tissue ablation indicate that large particles are ejected with high velocity from the irradiated tissue, which strongly supports the proposed mechanism of a water-mediated explosive process.

Several studies reported microleakage around composite restorations when cavity preparation was done or treated by Er:YAG laser ${ }^{10-14}$; however, there is no agreement between all experiments or researchers. Understanding how much dental tissue is in fact modified by this laser and how the laser could be affecting the interaction between the irradiated surface and the adhesive system constitutes is an important topic for clinicians. Because the Er:YAG laser is highly absorbed by water, the resultant irradiated surface can present dehydrated features. This may make it difficult for the adhesive agent to diffuse into the tissue.

Kataumi et al. 15 showed that dentin irradiated by Er:YAG laser does not present a smear layer; even smear plugs are absent. After the application of adhesive agent over irradiated dentin (with or without phosphoric acid etching), the so-called "plastic dentin" could not be observed, and the resin tags were thinner than those found in the other group (i.e., burr). Er:YAG laser irradiation produced an acid-resistant dentin, especially the peritubular dentin, which showed increased acid resistance

${ }^{1}$ Instituto de Física de São Carlos, USP, São Carlos, Brazil.

${ }^{2}$ Faculdade de Odontologia de Araraquara, UNESP, Brazil. 
when compared with the intertubulardentin. This study ${ }^{15}$ showed that Er:YAG laser irradiation affected the superficial dentin surface as well as the subsurface, to a depth of $20 \mu \mathrm{m}$.

Since there are no scientific reports about the use of rewetting in cavity preparation after Er:YAG irradiation, the present study considers different conditions of chemical treatmentsthat is, preparing enamel and dentin irradiated surfaces before composite resin restoration. We test a modified adhesive technique to improve bioattachment between enamel and dentin substrate after cavity preparation by Er:YAG laser and a onestep adhesive agent.

\section{MATERIALS AND METHODS}

\section{Teeth preparation}

In this study, we used 18 intact, recently extracted human third molar teeth. The teeth were obtained from a dental clinic with a protocol approved by an institutional review board. The teeth were kept in an appropriate environment (saline solution) to avoid dryness.

Teeth were randomly classified in five different groups in terms of treatment. Each group had seven samples. In all samples, cavities of class V were prepared, considering buccal and lingual surfaces. In groups I and II, we used a carbide burr; in groups III, IV, and V, the Er:YAG laser was used. The characteristics of each group were as follows: GI-cavity preparation, 37\% etching acid, adhesive agent, and composite; GII-cavity preparation, $37 \%$ etching acid, primer agent, adhesive agent, and composite; GIII-cavity preparation, adhesive agent, and composite resin; GIV-cavity preparation, $37 \%$ etching acid, adhesive agent, and composite resin; GVcavity preparation, $37 \%$ etching acid, primer agent, adhesive agent, and composite resin restoration.

The materials used were as follows: $37 \%$ phosphoric acid (3M, USA), adhesive agent Single Bond lot 1105 (3M, USA), primer agent Scotchbond Multi-Purpose 2 lot 3008 (3M, USA), and composite resin Filtek Z250 A2 lot 2004-07 (3M, USA). Each time that etching by phosphoric acid was used, the sample was washed and softly dry using an absorbed cone paper.

We chose a primer solution composed of 10\% HEMA (2-hydroxyethyl methacrylate) aqueous solution because it is capa- ble of re-expanding collapsed demineralized dentin effectively. Some hydrophilic monomers, such as HEMA, are extremely soluble in either water or acetone. If HEMA can replace water in the spaces around collagen fibrils, it can serve as a polymerizable solvent for subsequently placed adhesive monomers, given sufficient diffusion time. ${ }^{16}$

\section{Laser system}

The Er:YAG laser system we used was Twin Light (Fotona Medical Lasers, Slovenia) with $2940 \mathrm{~nm}$, peak energy of 500 $\mathrm{mJ}$, repetition rate of $2-15 \mathrm{~Hz}$, pulse width of $200-450 \mu \mathrm{sec}$, articulate arm, handpiece with sapphire window and noncontact, with spot size of $0.466 \mathrm{~mm}^{2}$.

\section{Experimental irradiation}

Teeth were exposed to focused Er:YAG laser with the following parameters: $300 \mathrm{~mJ}$ of energy per pulse, $10 \mathrm{~Hz}$ of repetition rate, $3.0 \mathrm{~W}$ of average power, during $90 \mathrm{sec}$ of exposition time, resulting in a fluence of $64.38 \times 10^{3} \mathrm{~J} / \mathrm{cm}^{2}$ and an intensity of $64.38 \times 10^{2} \mathrm{~W} / \mathrm{cm}^{2}$; using a carbide burr in a high-speed handpiece, cavities were prepared with an approximate volume of 1.0 $\times 2.0 \times 2.0 \mathrm{~mm}^{3}$ (width, length, and depth respectively), as shown in Figure 1. The oclusal wall was finished at the level of enamel, and the cervical wall was finished in dentin.

After the restorations were polished with gold (no. 3195F) and silver (no. 3195FF) diamond (KG Sorensen, Brazil) $24 \mathrm{~h}$ later, all samples were maintained in distilled water at $37^{\circ} \mathrm{C}$ for 7 days. Then, the samples were polished again, using sequential Sof Lex discs (3M, USA).

In order to proceed with the microleakage test, all restored teeth (except for the area of the filled cavity and an area 2.0 $\mathrm{mm}$ outside of the margins of the filled cavity) were completely coated with two layers of epoxy resin and one layer of nail varnish, and then subjected to the thermal cycling varying temperature in $10^{\circ} \mathrm{C}$ and $50^{\circ} \mathrm{C}$, for 500 cycles, for $15 \mathrm{sec}$ in each temperature per cycle. After thermal cycling, all samples were immersed in 50\% aqueous silver nitrate solution for $2 \mathrm{~h}$, followed by immersion in a photorevealing solution under fluorescent light for $16 \mathrm{~h}$.

After washing with water, the samples were bisected longitudinally using a carborundum disc and each part was coated in

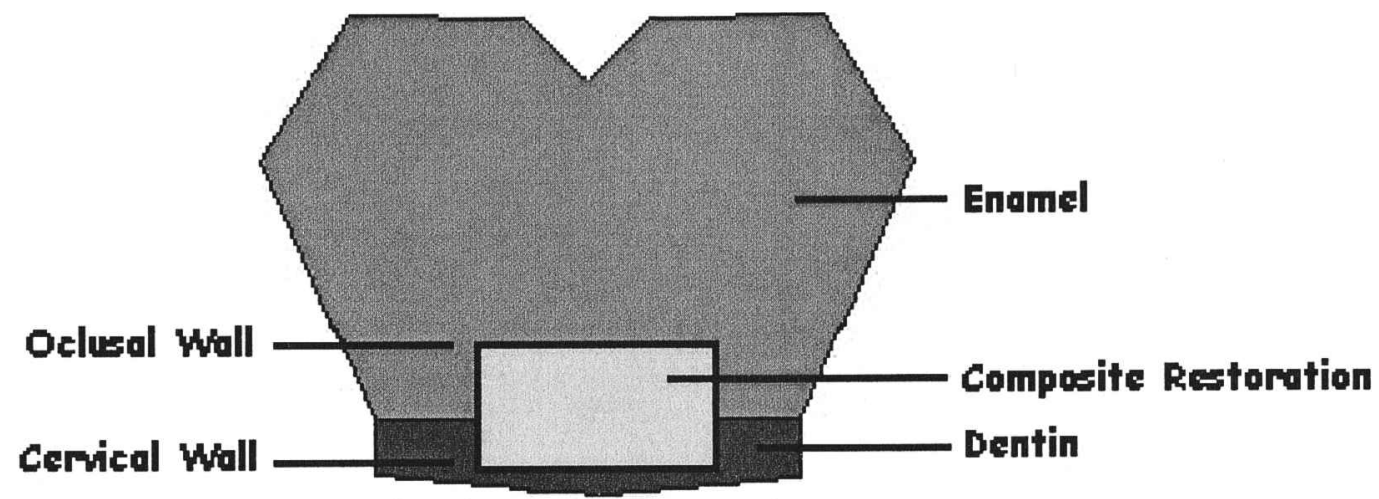

FIG. 1. Scheme of class V cavity preparation location. 
Table 1. Criterion for Degree of Microleakage

\begin{tabular}{ll}
\hline Score & \multicolumn{1}{c}{ Criterion } \\
\hline 0 & No dye penetration \\
1 & Dye penetration up to a third of the length of the wall \\
2 & Dye penetration between one and two thirds of the length of the wall \\
3 & Dye penetration beyond of two thirds of the length of the wall \\
\hline
\end{tabular}

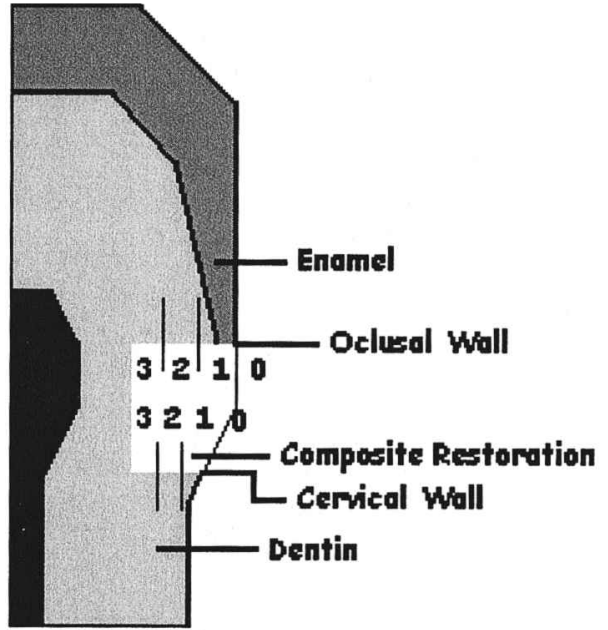

FIG. 2. Scheme of microleakage scores.

acrylic resin, individually. Each sample was sectioned, with one cut dividing the composite resin restoration in two parts. Both parts of each sample were evaluated under graduated magnification (times 40) to assess the degree of microleakage.

The degree of microleakage using the dye penetration method was scored in a blinded manner based on a four-grade scale as shown in Table 1 and Figure 2.

\section{RESULTS AND DISCUSSION}

Scores for each sample were analyzed using the following statistical tests: Mann-Whitney to compare oclusal and cervical walls and Kruskal-Wallis to compare the different treatments for each cavity preparation.

The Mann-Whitney test showed a statistically significant difference, at $1 \%(p=0.01)$. This means that at $99 \%$ probability, oclusal and cervical walls are not similar. This result supports the Kruskal-Wallis test considering different treatments and different walls.

The Kruskal-Wallis test showed a statistically significant difference at $1 \%(p=0.01)$, when all treatments were compared together - that is, all treatments for enamel and all treatments for dentin. In pairs, at $5 \%(p=0.05)$ as a significance level, some interesting findings were observed (Tables 2 and 3 ). Table 2 shows interactions of treatment for the oclusal wall, and Table 3 the same conditions for the cervical wall. Figures 3 and 4 show graphs for oclusal and cervical walls when the degree of microleakage is a function of the different treatments; all samples (seven in each group) are shown.

Evaluating the oclusal wall, in enamel substrate, there is a statistically significant difference between GI (burr + phosphoric acid + adhesive agent + composite) and GII (burr + phosphoric acid + primer + adhesive agent + composite) in comparison with all other groups: GIII (laser + adhesive agent + composite), GIV (laser + phosphoric acid etched + adhesive agent + composite), and GV (laser + phosphoric acid etched + primer + adhesive

Table 2. Kruskal-Wallis Statistical Test between Pairs of Samples, Considering Oclusal Wall

$($ SIGNIFICANCE LEVEL $=5 \%)$

Comparison between average of samples

\begin{tabular}{|c|c|c|c|c|c|}
\hline \multirow[b]{3}{*}{ Pairs of samples } & \multicolumn{4}{|c|}{ Comparison between average of samples } & \multirow[b]{3}{*}{ Significance } \\
\hline & \multirow[b]{2}{*}{ Difference between media } & \multicolumn{3}{|c|}{ Critical values $(p)$} & \\
\hline & & 0.05 & 0.01 & 0.001 & \\
\hline GIo $\times$ GIIo & 5,9286 & 7,2611 & 9,7786 & 12,9647 & ns \\
\hline GIo $\times$ GIIIo & 18,9286 & 7,2611 & 9,7786 & 12,9647 & $5 \%$ \\
\hline GIo $\times$ GIVo & 17,3571 & 7,2611 & 9,7786 & 12,9647 & $5 \%$ \\
\hline GIo $\times$ GVo & 9,1429 & 7,2611 & 9,7786 & 12,9647 & $5 \%$ \\
\hline GIIo $\times$ GIIIo & 13,0000 & 7,2611 & 9,7786 & 12,9647 & $5 \%$ \\
\hline GIIo $\times$ GIVo & 11,4286 & 7,2611 & 9,7786 & 12,9647 & $5 \%$ \\
\hline GIIo $\times$ GVo & 3,2143 & 7,2611 & 9,7786 & 12,9647 & ns \\
\hline GIIIo $\times$ GIVo & 1,5714 & 7,2611 & 9,7786 & 12,9647 & ns \\
\hline GIIIo $\times$ GVo & 9,7857 & 7,2611 & 9,7786 & 12,9647 & $5 \%$ \\
\hline GIVo $\times$ GVo & 8,2143 & 7,2611 & 9,7786 & 12,9647 & $5 \%$ \\
\hline
\end{tabular}


Table 3. Kruskal-Wallis Statistical Test between Pairs of Samples, Considering Cervical Wall $($ SIGNIFICANCE LEVEL $=5 \%)$

Comparison between average of samples

Critical values $(p)$

\begin{tabular}{|c|c|c|c|c|c|}
\hline \multirow[b]{2}{*}{ Pairs of samples } & \multirow[b]{2}{*}{ Difference between media } & & \multirow[b]{2}{*}{ Significance } \\
\hline & & 0.05 & 0.01 & 0.001 & \\
\hline GIc $\times$ GIIc & 3,2857 & 6,8153 & 9,1784 & 12,1688 & ns \\
\hline GIc $\times$ GIIIc & 17,3571 & 6,8153 & 9,1784 & 12,1688 & $5 \%$ \\
\hline GIc $\times$ GIVc & 13,0000 & 6,8153 & 9,1784 & 12,1688 & $5 \%$ \\
\hline $\mathrm{GIc} \times \mathrm{GVc}$ & 6,4286 & 6,8153 & 9,1784 & 12,1688 & $\mathrm{~ns}$ \\
\hline GIIc $\times$ GIIIc & 20,6429 & 6,8153 & 9,1784 & 12,1688 & $5 \%$ \\
\hline GIIc $\times$ GIVc & 16,2857 & 6,8153 & 9,1784 & 12,1688 & $5 \%$ \\
\hline GIIc $\times$ GVc & 9,7143 & 6,8153 & 9,1784 & 12,1688 & $5 \%$ \\
\hline GIIIc $\times$ GIVc & 4,3571 & 6,8153 & 9,1784 & 12,1688 & $\mathrm{~ns}$ \\
\hline GIIIc $\times$ GVc & 10,9286 & 6,8153 & 9,1784 & 12,1688 & $5 \%$ \\
\hline GIVc $\times$ GVo & 6,5714 & 6,8153 & 9,1784 & 12,1688 & $\mathrm{~ns}$ \\
\hline
\end{tabular}

agent + composite). These findings show that whatever treatment is used after cavity preparation with the Er:YAG laser, it is very difficult to find a degree of microleakage lower than or similar to that when the burr is used to prepare the enamel surface.

It is possible that the different interaction mechanisms of Er:YAG laser and phosphoric acid with the enamel surface. Phosphoric acid acts by removing minerals components, such as $\mathrm{Ca}$ (calcium) and $\mathrm{P}$ (phosphorus), elements of hydroxiapatite, and by the other side, Er:YAG laser ablates enamel removing water and phosphate groups, resulting in a surface rich in $\mathrm{Ca}$, however dehydrated. The adhesive agent used is hydrophilic and interacts with a hydrated surface indeed with some pore to permits micro-retentions through interpenetrations of this adhesive agent into enamel tissue.

Other findings showed a statistical similarity between GII (burr + phosphoric acid etched + primer + adhesive agent + composite) and GV (laser + phosphoric acid etched + primer + adhesive agent + composite), and a difference between GIV (laser + phosphoric acid etched + adhesive agent + composite) and GV (laser + phosphoric acid etched + primer + adhe-

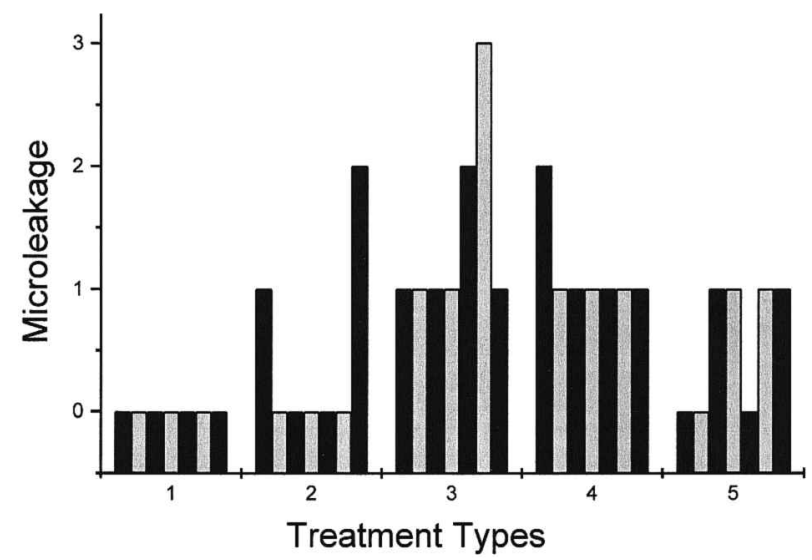

FIG. 3. Graph of all groups of treatment type as a function of microleakage scores, considering oclusal wall (enamel) $(1=$ GI; 2 = GII; 3 = GIII; $4=$ GIV; and $5=$ GV). sive agent + composite). The use of primer (10\% HEMA aqueous solution) application just after $37 \%$ phosphoric acid results in a microleakage showing similar degrees with the same primer was applied before the adhesive agent in cavities done by burr, it can show that primer is very important even for enamel surfaces after Er:YAG laser irradiation to prevent microleakage.

As statistical tests showed a significant difference between GIV and GV, this result confirms the important function of primer application just after Er:YAG laser irradiation in the enamel surface.

Considering dentin surface (cervical wall), the statistical similarity is present just between GI (burr + phosphoric acid + adhesive agent + composite) and GV (laser + phosphoric acid etched + primer + adhesive agent + composite). This indicates that cavities prepared with the Er:YAG laser need to be treated in advance with phosphoric acid and primer, even when using a one-step adhesive agent. It could be a result of the capability of Er:YAG laser in removing more water from a tissue with the use of water spray and more sensible to allow adhesive agent diffusion.

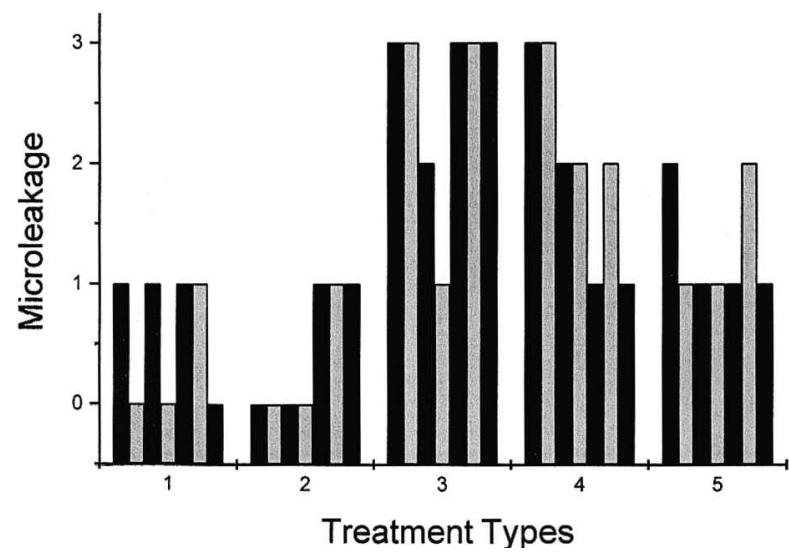

FIG. 4. Graph of all groups of treatment type as a function of microleakage scores, considering cervical wall (dentin) $(1=$ GI; 2 = GII; 3 + GIII; $4=$ GIV; and $5=$ GV). 
When the same conditions were repeated changing the cutting tool (burr or laser), there is no statistical similarity in the cervical wall, dentin as a substrate; GI (burr + phosphoric acid + adhesive agent + composite) was different from GIV (laser + phosphoric acid + adhesive agent + composite) and GII (burr + phosphoric acid + primer + adhesive agent + composite $)$ was different from GV (laser + phosphoric acid + primer + adhesive agent + composite), at the level of $5 \%$. It means that using primer (10\% HEMA aqueous solution) application just after $37 \%$ phosphoric acid results in a smaller microleakage, however Er:YAG laser can not replace phosphoric acid etching.

Er:YAG laser seems to affect bioattachment more in dentin surface than enamel surface, as we can see in Figures 3 and 4. Chemical composition could explain this fact; dentin has more water than enamel, so after irradiation with Er:YAG laser dentin becomes more affected than enamel, resulting in difficulty in promoting an adequate surface to allow diffusion of the adhesive agent.

\section{CONCLUSION}

In conclusion, we have presented a microleakage study considering a new topic: re-wetting of enamel and dentin surfaces irradiated by Er:YAG laser. Our results showed some steps towards the improvement of marginal adaptation of composite resin Class V restorations, when Er:YAG laser was used for cavity preparations:

1. Er:YAG laser cannot replace phosphoric acid etching.

2. Er:YAG laser seems to affect bioattachment more in dentin surface than in enamel surface.

3 . The application of primer solution (10\% hydroxyl ethyl methacrylate aqueous solution) as a re-wetting solution just after etching with $37 \%$ phosphoric acid results in smaller microleakage for enamel and dentin surfaces.

4. We recommend the use of this primer solution (10\% HEMA) after cavity preparation with the Er:YAG laser system.

\section{ACKNOWLEDGMENTS}

We acknowledge financial support from CNPq (PRONEX) and FAPESP (CEPID program). We also acknowledge L.T. Moriyama (IFSC-USP) and 3M, of Brasil (Sr. A. Andreazi Filho, Manager), for materials used in this work.

\section{REFERENCES}

1. Barnes, D.M., McDonald, N.J., Thompson, V.P., et al. (1994). Microleakage in facial and lingual class 5 composite restorations: a comparison. Oper. Dent. 19, 133-137.
2. Hibst, R., Keller, U., and Steiner, R. (1988). Die Wirkung gepulster Er:YAG-laserstrahlung auf zahngewebe. Laser Med. Surg. 4, 163-165.

3. Paghdiwala, A.F., Vaidyanathan, T.K., and Paghdiwala, M.F. (1993). Evaluation of erbium:YAG radiation of hard dental tissues analysis of temperature changes, depth of cuts and structural effects. Scanning Microsc. 7, 989-997.

4. Pelagalli, J., Gimbel, C.B., Hansen, R.T., et al. (1997). Investigation study of the use of Er:YAG laser versus dental drill for caries removal and cavity preparation-phase I. J. Clin. Laser Med. Surg. $15,109-116$.

5. Dostálová, T., Jelínková, H., Krejsa, O., et al. (1997). Dentin and pulp response to erbium:YAG laser ablation: a preliminary evaluation of human teeth. J. Clin. Laser Med. Surg. 15, 117122.

6. Tokonabe, H., Kouji, R., Watanabe, H., et al. (1999). Morphological changes of human teeth with Er:YAG laser irradiation. J. Clin. Laser Med. Surg. 17, 7-12.

7. Hadley, J., Young, D.A., Eversole, L.R., et al. (2000). A laser-powered hydrokinetic system for caries removal and cavity preparation. J. Am. Dent. Assoc. 131, 777-785.

8. Yamada, Y., Hossain, M., Nakamura, Y., et al. (2001). Removal of carious dentin by mechanical, chemomechanical and Er:YAG laser in deciduous teeth. J. Oral Laser Appl. 1, 109-114.

9. Fried, D. (2000). IR laser ablation of dental enamel. Proc. SPIE 3910, 136-148.

10. Wright, G.Z., McConnell, R.J., and Keller, U. (1993). Microleakage of class $\mathrm{V}$ composite restorations prepared conventionally and with Er:YAG laser: a pilot study. Pediatr. Dent. 15, 425-426.

11. Khan, M.F.R., Yonaga, K., Kimura, Y., et al. (1998). Study of microleakage at class I prepared by Er:YAG laser using three types of restorative materials. J. Clin. Laser Med. Surg. 16, 305-308.

12. Niu, W., Eto, J.N., Kimura, Y., et al. (1998). A study on microleakage after resin filling of class $\mathrm{V}$ cavities prepared by Er:YAG laser. J. Clin. Laser Med. Surg. 16, 227-231.

13. Lizarelli, R.F.Z., Kurachi, C., Porto Neto, S.T., et al. (2000). Comparative study in vitro of microleakage in class V cavity preparation with and without Er:YAG laser. Proc. SPIE 3910, 254-260.

14. Lizarelli, R.F.Z., Silva, P.C.G., Kurachi, C., et al. (2002). Estudopiloto comparativo da microinfiltração in vitro entre preparos cavitários classe $\mathrm{V}$, através de ponta diamantada em alta rotação ou laser de Er:YAG seguido ou não de ataque ácido. J. Brasil. Dent. 1, $33-41$.

15. Kataumi, M., Nakajima, M., Yamada, T., et al. (1998). Tensile bond strength and SEM evaluation of Er:YAG laser irradiated dentin using dentin adhesive. Dent. Mater. J. 17, 125-138.

16. Nakabayashi, N., and Pashley, D.H. (1998). Hybridization of dental hard tissues. Tokyo: Quintessence.

Address reprint requests to: Rosane F.Z. Lizarelli, D.D.S., M.Sc., Ph.D. Instituto de Física de São Carlos-USP P.O. Box 369 CEP 13560-900 São Carlos, SP, Brasil

E-mail: lizarelli@if.sc.usp.br 